OUTP-95-62 S, hep-th/9511134

\title{
Logarithmic Operators and Hidden Continuous Symmetry in Critical Disordered Models
}

\author{
J.-S. Caux, Ian I. Kogan and A. M. Tsvelik \\ Department of Physics, University of Oxford, 1 Keble Road, \\ Oxford, OX1 3NP, UK
}

(July 10, 2021)

\begin{abstract}
We study the model of $(2+1)$-dimensional relativistic fermions in a random non-Abelian gauge potential at criticality. The exact solution shows that the operator expansion contains a conserved current - a generator of a continuous symmetry. The presence of this operator changes the operator product expansion and gives rise to logarithmic contributions to the correlation functions at the critical point. We calculate the distribution function of the local density of states in this model and find that it follows the famous log-normal law.
\end{abstract}

Typeset using REVTEX 


\section{INTRODUCTION}

In this paper we continue to study the model of $(2+1)$-dimensional massless Dirac fermions interacting with a random static non-Abelian gauge potential. The Hamiltonian (or rather a generating functional for the Green's functions) of this model is given by

$\mathrm{i} \hat{H}-\epsilon_{n} \hat{I}=\int \mathrm{d}^{2} x\left(R_{\alpha}^{+}, L_{\alpha}^{+}\right)\left(\begin{array}{cc}\left(\partial_{x}-\mathrm{i} \partial_{y}\right) \delta_{\alpha \beta}-\mathrm{i} A_{\alpha \beta}^{+}(x, y) & -\epsilon_{n} \\ -\epsilon_{n} & \left(\partial_{x}+\mathrm{i} \partial_{y}\right)-\mathrm{i} A_{\alpha \beta}^{-}(x, y)\end{array}\right)\left(R_{\beta}, L_{\beta}\right)$

where the random fields $A_{\alpha \beta}^{a}(x, y)(a= \pm)$ have a Gaussian distribution:

$$
\left\langle A_{\alpha \beta}^{a}\left(\vec{r}_{1}\right) A_{\gamma \eta}^{b}\left(\vec{r}_{2}\right)\right\rangle=\mathcal{A} \delta\left(\vec{r}_{1}-\vec{r}_{2}\right) \delta_{a,-b} \delta_{\alpha \eta} \delta_{\beta \gamma}
$$

The fermionic fields $R_{\alpha}, L_{\alpha}$ represent respectively the right and the left moving components of the spinor field, and $\alpha$ takes the values $1, \ldots, N$. This model was introduced in Ref. [1] to describe normal excitations in two dimensional non-s-wave superconductors with disorder. In this context $N$ denotes the number of nodes of the order parameter on the Fermi surface.

Since the disorder is time-independent we consider the Fourier components of the fermionic fields with different frequencies separately. This reduces the dimensionality of the problem, making it two dimensional. The model (1) is exactly solvable in the subspace $\epsilon=0$, where it is critical. The presence of the superconducting order parameter fixes the chemical potential and thus insures that the disorder is diagonal in chirality. This is essential for the criticality at $\epsilon=0$. At the critical point, one can apply the methods of conformal field theory and calculate scaling dimensions of the fields and of their multi-point correlation functions. This gives us a rare opportunity to obtain nonperturbative results for a non-trivial random theory. We hope that a study of this exact solution will give an insight into general properties of random systems.

The averaging over the disorder can be done either through the replica trick [2] or using the supersymmetric approach [3], [4]. In this paper we shall mostly use the replica approach with which we are more familiar. To demonstrate that the two approaches are equivalent we 
shall (i) compare the conformal dimensions of the primary fields and (ii) demonstrate that both representations give the same conformal blocks for the four-point correlation function.

As in the standard localization theory (see for example [5]), one can integrate out the fast degrees of freedom and derive an effective action for the slow ones in the form of a sigma model. This program was carried out in Ref. [1]. The resulting sigma model has the following action:

$$
S=S_{0}+M \epsilon_{n} \int \mathrm{d}^{2} x \operatorname{Tr}\left(Q+Q^{+}\right)
$$

where the $S_{0}$ action contains the Wess-Zumino term:

$$
\begin{gathered}
S_{0}=\frac{N r}{2} \int \mathrm{d}^{2} x\left(\partial_{\mu} \Phi\right)^{2}+N W[S U(r) ; g] \\
W[S U(2 r) ; g]=\frac{1}{16 \pi} \int \mathrm{d}^{2} x\left[\operatorname{Tr}\left(\partial_{\mu} g^{+} \partial_{\mu} g\right)+\frac{2}{3} \int_{0}^{\infty} \mathrm{d} \xi \epsilon_{a b c} \operatorname{Tr}\left(g^{+} \partial_{a} g g^{+} \partial_{b} g g^{+} \partial_{c} g\right)\right]
\end{gathered}
$$

where $Q$ is the $r \times r$ matrix $Q=g \exp [\mathrm{i} \sqrt{4 \pi} \Phi]$, and where $g$ belongs to the $\mathrm{SU}(\mathrm{r})$ group. $\Phi$ is a real scalar field defined on the cirle with circumference $\sqrt{\pi}$. The quantity $M$ is the energy scale introduced by the disorder: $M \sim \exp [-2 \pi / N \mathcal{A}]$; it marks the crossover from the bare density of states $(\operatorname{DOS}) \rho(\epsilon) \sim|\epsilon|$ to the renormalized DOS $\rho(\epsilon) \sim|\epsilon|^{\nu}\left(\nu=\left(2 N^{2}-1\right)^{-1}\right)$. $M$ serves as the ultraviolet cut-off for the sigma model (4).

It is well known that in conventional localization theories the average DOS is not affected by the disorder (its higher moments are, however). It is not the case for the model (1), where the DOS is directly proportional to the order parameter. As it was shown in [1], the local DOS is given by

$$
\rho(\epsilon, x)=\frac{M}{r} \operatorname{Tr}\left[Q(x)+Q^{+}(x)\right]
$$

This means that even the average DOS is strongly renormalized. At $\epsilon=0$ the sigma model (四) is critical and $\langle\rho(0, x)\rangle=0$, as it might be expected in a critical theory in two dimensions [6]. In three dimensions a finite DOS emerges at $\epsilon=0$ [7]. In our previous publications we interpreted this effect as a manifestation of violation of some continuous symmetry present in the theory ( [1], [2]). However, the meaning of this symmetry has remained obscure. In this paper we identify the operators which generate this symmetry and derive their algebra. 
The paper is organized as follows. In Section II we discuss general properties of the model at criticality and derive the expression for the conformal dimensions of its primary fields. In Section III we derive the expression for the four-point correlation function of local DOS. It turns out that this correlation function contains logarithmic singularities and therefore a fusion of local DOS generates operators with unusual properties - the so-called logarithmic operators. In Section IV we develop a general theory of such operators and demonstrate that the appearance of such operators always implies the presence of some continuous symmetry. It may well be that this symmetry is present in all critical models with disorder. In Section V we show how these general results hold for the model (4). In Section VI we study correlation functions in the model deformed by the logarithmic operators away from criticality. It turns out that even in the case of a marginal deformation the correlation functions have logarithmic corrections. In Section VII we derive the log-normal distribution function of the local DOS. The paper contains a conclusion and an appendix where the conformal blocks of replica and supersymmetric theories are compared.

\section{CONFORMAL DIMENSIONS}

The WZNW model has been well studied at finite $r$. There is an extensive literature on the subject, but we particularly recommend the original publication by Knizhnik and Zamolodchikov [12]. These authors derived explicit expressions for the four-point correlation functions of primary fields which we are going to exploit. In our calculational procedure we follow the general principle: when calculating any $n$-point correlation function $F_{r}(1,2, \ldots n)$ $r$ is treated as an arbitrary number on all intermediate steps of the calculations until the final expression is obtained. We define the replica limit as follows:

$$
F(1,2, \ldots n)=\lim _{r \rightarrow 0} \frac{2 N}{r} F_{r}(1,2, \ldots n)
$$

The reason for the introduction of the extra factor $2 N$ will be discussed later.

Let us study the correlation functions of the $Q, Q^{+}$-fields. The problem of indices is simplified by the fact that the $Q_{p q}$ matrices are slow parts of the operators 


$$
Q_{p q} \sim \sum_{\alpha=1}^{N} R_{\alpha, p}^{+} L_{\alpha, q}
$$

From this fact one can derive a simple recipe for the index structure of $n$-point correlation functions: it is the same as for the $n$-point function of the $\sum_{\alpha} R_{\alpha, p}^{+} L_{\alpha, q}$-fields in the theory of massless free fermions. The simplest example is the 2-point function [2], [3]:

$$
\left\langle Q_{p_{1} q_{1}}(z, \bar{z}) Q_{q_{2} p_{2}}^{+}(0,0)\right\rangle=\delta_{q_{1} q_{2}} \delta_{p_{1} p_{2}} \frac{1}{(M|z|)^{2 / N^{2}}}
$$

where $1 / 2 N^{2}$ is the conformal dimension of the composite operator $Q$ given by the sum of the dimensions of the bosonic exponent $\exp [\mathrm{i} \sqrt{4 \pi} \Phi]$ and of the operator field $g_{p r}$ from the fundamental representation of the $\mathrm{SU}(\mathrm{r})$ group:

$$
\Delta=\lim _{r \rightarrow 0}\left[\frac{1}{2 r N}+\frac{(r-1 / r)}{2(N+r)}\right]=\frac{1}{2 N^{2}}
$$

In the replica limit we get from Eq.(8)

$$
G(z, \bar{z}) \equiv \lim _{r \rightarrow 0}(2 N / r)\left\langle\operatorname{Tr}[Q(z, \bar{z})] \operatorname{Tr}\left[Q^{+}(0,0)\right]\right\rangle=(M|z|)^{-2 / N^{2}}
$$

All other operators are generated by fusion of the fundamental fields $\operatorname{Tr} Q$ and $\operatorname{Tr} Q^{+}$. The corresponding primary fields are composite fields of bosonic exponents and Wess-Zumino tensors belonging to irreducible representations of the SU(r) group. These representations are classified by Young tableaus which can be represented by a string of numbers $f_{1}>f_{2}>$ $\ldots>f_{r} \geq 0$. Only representations with $f_{l} \leq N-1$ are generated [12]. The corresponding conformal dimensions are given by the expressions

$$
\begin{aligned}
\Delta_{f} & =\frac{C_{f}}{N+r}+\frac{f^{2}}{2 r N} \\
C_{f} & =\frac{1}{2} \sum_{l}\left[f_{l}^{2}+(r+1-2 l) f_{l}\right]-\frac{f^{2}}{2 r}
\end{aligned}
$$

where $f=\sum_{l} f_{l}$. In the replica limit we get

$$
\Delta_{f}=\frac{f^{2}}{2 N^{2}}+\frac{1}{2 N} \sum_{l}\left[f_{l}^{2}-(2 l-1) f_{l}\right]
$$


This expression coincides with the conformal dimensions obtained by the supersymmetric approach after rows and columns in the Young tableau are interchanged. For in-

stance, for the representation $(\overbrace{1,1, \ldots 1}^{m}, 0, .$.$) we reproduce the expression obtained in [3] (see$ Eq.(4.48b)there) for the $(m, 0, \ldots 0)$ representation:

$$
\Delta_{m}=\frac{m}{2 N}\left[1-\frac{(N-1) m}{N}\right]
$$

\section{FOUR POINT CORRELATION FUNCTION OF THE ORDER PARAMETER FIELDS}

Let us now study the four point correlation function of the $Q, Q^{+}$fields. The index structure is the same for all $r$ [12]:

$$
\begin{aligned}
\left\langle Q_{p_{1} q_{1}}\left(z_{1}, \bar{z}_{1}\right) Q_{q_{2} p_{2}}^{+}\left(z_{2}, \bar{z}_{2}\right)\right. & \left.Q_{p_{3} q_{3}}\left(z_{3}, \bar{z}_{3}\right) Q_{q_{4} p_{4}}^{+}\left(z_{4}, \bar{z}_{4}\right)\right\rangle \\
& =M^{-4 / N^{2}}\left[\frac{\left|z_{13} z_{24}\right|}{\left|z_{12} z_{14} z_{23} z_{34}\right|}\right]^{2 / N^{2}}(W+\tilde{W}), \\
\tilde{W} & =\left[\delta_{p_{1} p_{2}} \delta_{p_{3} p_{4}} \delta_{q_{1} q_{2}} \delta_{q_{3} q_{4}} W_{11}(x, \bar{x})+\delta_{p_{1} p_{3}} \delta_{p_{2} p_{4}} \delta_{q_{1} q_{3}} \delta_{q_{2} q_{4}} W_{22}(x, \bar{x})\right], \\
W & =\left[\delta_{p_{1} p_{2}} \delta_{p_{3} p_{4}} \delta_{q_{1} q_{3}} \delta_{q_{2} q_{4}} W_{12}(x, \bar{x})+\delta_{p_{1} p_{3}} \delta_{p_{2} p_{4}} \delta_{q_{1} q_{2}} \delta_{q_{3} q_{4}} W_{21}(x, \bar{x})\right]
\end{aligned}
$$

where

$$
x=\frac{z_{12} z_{34}}{z_{13} z_{24}}, \bar{x}=\frac{\bar{z}_{12} \bar{z}_{34}}{\bar{z}_{13} \bar{z}_{24}}
$$

Here the functions $W_{A B}(x, \bar{x}),(A, B=1,2)$ satisfy linear differential equations (the Knizhnik-Zamolodchikov equations) which we shall discuss later in detail. Now note that in our theory we shall deal only with correlation functions of $\operatorname{Tr} Q, \operatorname{Tr} Q^{+}$(for simplicity we do not consider transport phenomena which would need introduction of advanced and retarded correlation functions). Since all correlation functions must be proportional to $r$, only the $W$ term (that is the term with all indices equal) survives in the replica limit, the $\tilde{W}$ term being proportional to $r^{2}$. Therefore we have 


$$
\begin{aligned}
& (2 N / r)\left\langle\operatorname{Tr} Q(1) \operatorname{Tr} Q^{+}(2) \operatorname{Tr} Q(3) \operatorname{Tr} Q^{+}(4)\right\rangle \\
& \quad=2 N M^{-4 / N^{2}}\left[\frac{\left|z_{13} z_{24}\right|}{\left|z_{12} z_{34} z_{23} z_{34}\right|}\right]^{2 / N^{2}}\left[W_{12}(x, \bar{x})+W_{21}(x, \bar{x})\right]
\end{aligned}
$$

The functions $W_{A B}(x, \bar{x})=U^{p q} W_{A}^{(p)}(x) W_{B}^{(q)}(\bar{x})$ are composed of linearly independent solutions of the Knizhnik-Zamolodchikov equation, written $W_{A}^{(p)}(x)$ and $W_{B}^{(q)}(\bar{x})$ (conformal blocks). In the replica limit these equations have the following form:

$$
N x \frac{d W_{1}}{d x}=-W_{2}, \quad N(1-x) \frac{d W_{2}}{d x}=W_{1}
$$

Thus for the function $W_{1}$ we get the following hypergeometric equation:

$$
N^{2} \frac{d}{d x}\left(x \frac{d W_{1}}{d x}\right)+\frac{W_{1}}{1-x}=0
$$

Here we encounter a problem. A hypergeometric equation has always two linearly independent solutions, normally expressed in terms of powers and hypergeometric functions. Usually there are three sets of solutions defined in the vicinity of $x=0,1$ and $\infty$ respectively. These pairs of solutions are related to each other via simple transformation rules (see, for example [13]). Eq.(20) is an exlusion: in the vicinity of $x=0$ one of the solutions contains a logarithmic singularity and cannot be expressed in terms of hypergeometric functions (this second solution was overlooked in the previous publication of one of the authors [2]):

$$
\begin{aligned}
W_{1}^{(0)}(x) & =F(1 / N,-1 / N, 1, x), \\
W_{1}^{(1)}(x) & =\ln x W_{1}^{(0)}(x)+H_{1}(x) \\
N W_{2}^{(0)}(x) & =x F(1+1 / N, 1-1 / N, 2, x), \\
N W_{2}^{(1)}(x) & =\ln x W_{2}^{(0)}(x)-N^{2}+H_{2}(x)
\end{aligned}
$$

where $H_{1,2}(x)$ are functions that are regular at $x=0$ :

$$
\begin{aligned}
& H_{1}(x)=\sum_{n=1}^{\infty} \frac{x^{n}(1 / N)_{n}(-1 / N)_{n}}{(n !)^{2}}[\psi(1 / N+n)-\psi(1 / N)+ \\
&+\psi(-1 / N+n)-\psi(-1 / N)-2 \psi(n+1)+2 \psi(1)] \\
& H_{2}(x)=x \sum_{n=0}^{\infty} \frac{x^{n}(1+1 / N)_{n}(1-1 / N)_{n}}{n !(n+1) !}[\psi(1+1 / N+n)-\psi(1+1 / N)+ \\
&\quad+\psi(1-1 / N+n)-\psi(1-1 / N)-\psi(n+1)+\psi(1)-\psi(n+2)+\psi(2)]
\end{aligned}
$$


where $(a)_{n}=\Gamma(a+n) / \Gamma(a)$. Only in the vicinity of $x=\infty$ are the solutions still hypergeometric functions (for $N \neq 2$ ).

At $|x|<<1$ we have

$$
\begin{aligned}
W_{1}^{(0)}(x)=1+O(x), & W_{1}^{(1)}(x)=\ln x[1+O(x)], \\
N W_{2}^{(0)}(x)=x+O\left(x^{2}\right), & N W_{2}^{(1)}(x)=-N^{2}+x \ln x[1+O(x)]
\end{aligned}
$$

Now we have to choose the matrix $U^{p q}$ in such a way that the resulting expression for the four point correlation function be a uniquely defined function in the complex plane of $x$. It also must be invariant under the permutation of points 1 and 3 (2 and 4$)$ which means the invariance under $x \rightarrow 1-x, \bar{x} \rightarrow 1-\bar{x}$ (crossing symmetry). These properties are achieved when

$$
U^{(01)}=U^{(10)}, U^{(11)}=0, U^{(00)}=h U^{(01)}
$$

To find $h$, we first note that the solutions to the Knizhnik-Zamolodchikov equations obey the monodromy properties

$$
\begin{aligned}
& W_{1}^{(0)}(1-x)=a_{i} W_{2}^{(i)}(x) \\
& W_{2}^{(0)}(1-x)=b_{i} W_{1}^{(i)}(x)
\end{aligned}
$$

where

$$
\begin{aligned}
& a_{0}=a_{1}[\psi(1 / N)+\psi(-1 / N)-\psi(2)-\psi(1)] \\
& a_{1}=b_{1}=\frac{N}{\Gamma(1 / N) \Gamma(-1 / N)} \\
& b_{0}=a_{1}[\psi(1 / N)+\psi(-1 / N)-2 \psi(1)]
\end{aligned}
$$

Using the crossing symmetry, we then find that

$$
h=\frac{a_{0} b_{1}+a_{1} b_{0}}{a_{1} b_{1}}=1 / 2
$$

Thus we get 


$$
\begin{aligned}
G(1,2,3,4)= & -\frac{1}{2 N}\left[\frac{\left|z_{13} z_{24}\right| M^{-2}}{\left|z_{12} z_{14} z_{23} z_{34}\right|}\right]^{2 / N^{2}} \\
& \times\left[W_{1}^{(0)}(x) W_{2}^{(1)}(\bar{x})+W_{1}^{(1)}(x) W_{2}^{(0)}(\bar{x})+\frac{1}{2} W_{1}^{(0)}(x) W_{2}^{(0)}(\bar{x})+(x \rightarrow \bar{x})\right]
\end{aligned}
$$

Here we choose $U^{(01)}=-1 / 4 N^{2}$ for normalization.

In order to derive the operator algebra of the model we consider various limits of this formula. In the limit $z_{43}=\epsilon \rightarrow 0$ we get

$$
\left\langle\left[Q Q^{+}(3)\right] Q(1) Q^{+}(2)\right\rangle=\frac{1}{\left|\epsilon z_{12}\right|^{2 / N^{2}}}\left[1-\frac{1}{N^{2}}\left(\frac{\epsilon z_{12}}{z_{13} z_{23}}+c . c\right) \ln \left(|\epsilon|\left|\frac{z_{12}}{z_{13} z_{23}}\right|\right)+\ldots\right]
$$

From now on we shall use $Q$ without subscripts instead of $\operatorname{Tr} Q$ assuming that the replica limit has been taken. We shall also put $M=1$.

The three-point correlation function (29) is very unusual from the conformal field theory point of view because it contains logarithms. Therefore we pause to consider general properties of logarithmic operators.

\section{GENERAL PROPERTIES OF LOGARITHMIC OPERATORS}

So far correlation functions with logarithms at criticality have been obtained in the WZNW model on the supergroup $G L(1,1)[8]$, in the $\mathrm{C}=-2$ model [9] and in gravitationally dressed CFT [10]. It was first pointed out by Gurarie in Ref. [9] that the appearence of logarithms in correlation functions is due to the presence of special operators, whose operator product expansions (OPE's) display logarithmic short-distance singularities. These

logarithmic operators have conformal dimensions degenerate with those of the usual primary operators, and it is this degeneracy that is at the origin of the logarithms (cf. our discussion of the degenerate hypergeometric equation in the previous Section). As a result of this degeneracy one can no longer completely diagonalize the Virasoro operator $L_{0}$, and the new operators together with the standard ones form the basis of the Jordan-cell for $L_{0}$. In order to get a better insight in the situation, we shall consider the simplest example, which was mentioned in [10], namely, the Liouville model with the action 


$$
S=\frac{1}{8 \pi} \int \mathrm{d}^{2} \xi \sqrt{g(\xi)}\left[\partial_{\mu} \phi(\xi) \partial^{\mu} \phi(\xi)+Q R^{(2)}(\xi) \phi(\xi)\right]
$$

$\left(R^{(2)}\right.$ is the Riemann curvature on a two-dimensional surface - the world sheet) with the stress-energy tensor

$$
T=-\frac{1}{2} \partial_{z} \phi \partial_{z} \phi+\frac{Q}{2} \partial_{z}^{2} \phi
$$

and the central charge

$$
\mathcal{C}=1+3 Q^{2}
$$

The primary field $\exp (\alpha \phi)$ has a dimension

$$
\Delta_{\alpha}=\alpha(Q-\alpha) / 2
$$

This means that there are two operators with the same dimension $\Delta_{\alpha}$, namely $V_{ \pm}=$ $\exp \left(\alpha_{ \pm} \phi\right)$, where

$$
\alpha_{ \pm}=\frac{Q}{2} \pm \frac{1}{2} \sqrt{Q^{2}-8 \Delta_{\alpha}}
$$

If $Q^{2}=8 \Delta_{\alpha}$, i.e. when $\alpha=Q / 2$, there is a degeneracy $\alpha_{+}=\alpha_{-}$and instead of two exponential primary fields we have only one exponent $C=\exp \left(\frac{1}{2} Q \phi\right)$ and the new operator $D=\phi \exp \left(\frac{1}{2} Q \phi\right)$ with the same dimension $\Delta=Q^{2} / 8$. The latter field is sometimes called the puncture operator. It was discussed in [11] in the context of the Liouville gravity, when the action (30) describes the gravitational (Liouville) sector of a (non)critical string in the conformal gauge.

It is easy to get the OPE of the stress-energy tensor $T$ with these fields. After simple calculations we find

$$
\begin{aligned}
& T(z) C(0)=\frac{\Delta}{z^{2}} C(0)+\frac{1}{z} \partial_{z} C(0)+\ldots \\
& T(z) D(0)=\frac{\Delta}{z^{2}} D(0)+\frac{1}{z^{2}} C(0)+\frac{1}{z} \partial_{z} D(0)+\ldots
\end{aligned}
$$

where the dimension of the fields $C$ and $D$ is $\Delta=Q^{2} / 8$ and the normalization of the field $D$ was defined as $D=(2 / Q) \phi \exp \left(\frac{1}{2} Q \phi\right)$. 
It is easy to see indeed that there is a mixing between $C$ and $D$ and that the Virasoro operator $L_{0}$ which is defined through the Laurent expansion $T(z)=\sum_{n} L_{n} z^{-n-2}$ is not diagonal

$$
L_{0}|C>=\Delta| C>, \quad L_{0}|D>=\Delta| D>+\mid C>
$$

Let us also note that usually one can think about factorization of the primary field into the product of chiral left and right operators using the decomposition $\phi(z, \bar{z})=\phi_{L}(z)+\phi_{R}(\bar{z})$, leading to $\exp [\alpha \phi]=\exp \left[\alpha \phi_{L}(z)\right] \times \exp \left[\alpha \phi_{R}(\bar{z})\right]$. For a logarithmic operator we have

$$
\phi \exp [\alpha \phi]=\phi_{L}(z) \exp \left[\alpha \phi_{L}(z)\right] \times \exp \left[\alpha \phi_{R}(\bar{z})\right]+\phi_{R}(\bar{z}) \exp \left[\alpha \phi_{L}(z)\right] \times \exp \left[\alpha \phi_{R}(\bar{z})\right]
$$

and thus the logarithmic operator is the sum of left and right operators each of which can be factorized.

This simple example illustrates a quite general property of all theories with logarithmic operators and OPE (33) is valid in all these theories. One can also obtain some general information about two- and three-point correlation functions with operators $C$ and $D$ starting from the four-point correlation function [9]

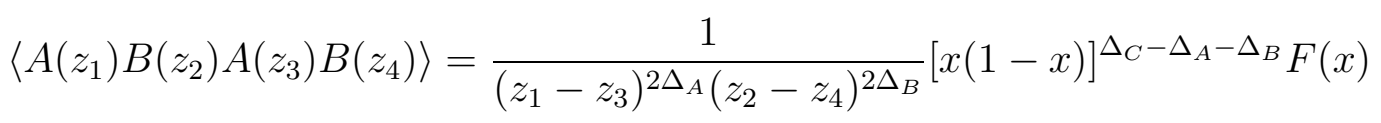

where $x$ is defined by Eq.(17), and where we have extracted the factor $[x(1-x)]^{\Delta_{C}-\Delta_{A}-\Delta_{B}}$ to make $F(x)$ finite at $x \rightarrow 0$ or $x \rightarrow 1$ in the ordinary case. In the case of logarithmic operators one will get $F(x)=(d+c \ln x+o(x))$ at small $x$. To reproduce the logarithmic singularity at $x=0$ after the fusion of $A\left(z_{1}\right)$ and $B\left(z_{2}\right)$ one has to postulate the following OPE (we restrict ourselves to the chiral sector):

$$
A\left(z_{1}\right) B\left(z_{2}\right)=\left(z_{1}-z_{2}\right)^{\Delta_{C}-\Delta_{A}-\Delta_{B}}\left[D+C \ln \left(z_{1}-z_{2}\right)+. .\right]
$$

Taking the limit $z_{1} \rightarrow z_{2}$ one immediately gets from the four-point correlation function the following three-point correlation functions:

$$
\begin{aligned}
& \left\langle C\left(z_{1}\right) A\left(z_{3}\right) B\left(z_{4}\right)\right\rangle=\frac{c}{z_{13}^{\Delta_{A}+\Delta_{C}-\Delta_{B}} z_{14}^{\Delta_{B}+\Delta_{C}-\Delta_{A}} z_{34}^{\Delta_{A}+\Delta_{B}-\Delta_{C}}} \\
& \left\langle D\left(z_{1}\right) A\left(z_{3}\right) B\left(z_{4}\right)\right\rangle=\frac{1}{z_{13}^{\Delta_{A}+\Delta_{C}-\Delta_{B}} z_{14}^{\Delta_{B}+\Delta_{C}-\Delta_{A}} z_{34}^{\Delta_{A}+\Delta_{B}-\Delta_{C}}}\left(c \ln \frac{z_{3}-z_{4}}{\left(z_{1}-z_{3}\right)\left(z_{1}-z_{4}\right)}+d\right)
\end{aligned}
$$


Now let us consider the $A\left(z_{3}\right)$ and $B\left(z_{4}\right)$ fusion which after insertion of (36) into (37) will lead to the following two-point correlation functions:

$$
\begin{aligned}
& \langle C(x) D(y)\rangle=\langle C(y) D(x)\rangle=\frac{c}{(x-y)^{2 \Delta_{C}}} \\
& \langle D(x) D(y)\rangle=\frac{1}{(x-y)^{2 \Delta_{C}}}(-2 c \ln (x-y)+d) \\
& \langle C(x) C(y)\rangle=0
\end{aligned}
$$

The first equation imposes a strong constraint on the dimensions $\Delta_{C}$ of logarithmic operators, namely that the dimension $\Delta_{C}$ must be an integer. To prove it let us note that we have $\langle C(x) D(y)\rangle=\langle C(y) D(x)\rangle$, which means that the correlation function is invariant under the permutation of $x$ and $y$, which means that $(-1)^{2 \Delta_{C}}=1$ so $\Delta_{C}=n$. In case of noninteger $\Delta_{C}$ one must have the structure constant $c=0$ and only the operator $D$ will survive in OPE (36), however in this case it will be an ordinary, nonlogarithmic operator.

This new result about dimensions of logarithmic operators means that for any logarithmic operator we have a hidden continous symmetry. This symmetry is generated by the conserved holomorphic (or antiholomorphic) current $C(z)$. This current is a symmetric tensor of rank $\Delta_{C}$, which is a usual vector current if $\Delta_{C}=1$. In the next Section we shall demonstrate the existence of such a conserved vector current in the model with disorder that we are considering. Let us note that we have also proved that there is no central extension in the corresponding current algebra, or in other words there is no anomalous Schwinger term in the current-current commutator. This is the direct consequence of the triviality of the correlation function $\langle C(z) C(0)\rangle=0$.

\section{OPERATOR PRODUCT EXPANSIONS IN THE MODEL (4)}

In this Section we demonstrate how the general theory just discussed applies to the model (4). For this end we study OPE's in this theory after the replica limit has been taken. In doing so we assume that the replica limit can be described by some quantum field theory. Here we encounter a certain ambiguity, namely, that we can define the correlation functions 
with an arbitrary prefactor. It turns out that this prefactor is fixed by the requirement of self-consistency of OPE's. The latter is achieved when one uses the definition (6). This explains the necessity of the factor $2 N$ in Eq.(6).

We suggest the following OPE:

$$
Q(z) Q^{+}(0)=|z|^{-2 / N^{2}}\left\{I-z\left[D(0)+C(0) \ln |z|^{2}\right]-\bar{z}\left[\bar{D}(0)+\bar{C}(0) \ln |z|^{2}\right] \ldots\right\}
$$

where $D, C$ and $\bar{D}, \bar{C}$ are some new operators whose correlation functions are to be found. Notice that in the conventional WZNW theory this operator expansion would contain the unit operator and the operator in the adjoint representation. However, the latter one has the conformal dimensions which vanish in the replica limit:

$$
\Delta_{a d}=\bar{\Delta}_{a d}=\frac{c_{v}}{c_{v}+N}=\frac{r}{r+N} \rightarrow 0
$$

Therefore we have here a situation described in the previous Section: the conformal dimensions of descendants of the unity operator become degenerate with the dimensions of descendants of some other primary field (the adjoint operator) which gives rise to logarithms.

Substituting (39) into Eq.(29) we get

$$
\begin{aligned}
& \left\langle Q(1) Q^{+}(2) C(3)\right\rangle=\frac{1}{2 N^{2}}\left|z_{12}\right|^{-2 / N^{2}} \frac{z_{12}}{z_{13} z_{23}} \\
& \left\langle Q(1) Q^{+}(2) D(3)\right\rangle=N^{-2}\left|z_{12}\right|^{-2 / N^{2}} \frac{z_{12}}{z_{13} z_{23}} \ln \left|\frac{z_{12}}{z_{13} z_{23}}\right|
\end{aligned}
$$

Setting $z_{12}=\epsilon$ in these equations and using the OPE (39) we get the following set of two-point correlation functions:

$$
\begin{aligned}
\langle D(1) C(2)\rangle & =-\frac{1}{2 N^{2} z_{12}^{2}}, \\
\langle C(1) C(2)\rangle & =0 \\
\langle D(1) D(2)\rangle & =\frac{2 \ln \left|z_{12}\right|}{N^{2} z_{12}^{2}}
\end{aligned}
$$

There are similar expressions for $\bar{C}, \bar{D}$-operators with $z$ being substituted for $\bar{z}$. Correlators of $C, D$ and $\bar{C}, \bar{D}$ are equal to zero.

Setting $z_{31}=\epsilon$ in Eqs.(41) we deduce the following OPE: 


$$
\begin{aligned}
C(z) Q(0) & =\frac{1}{2 N^{2} z} Q(0)+\ldots \\
D(z, \bar{z}) Q(0) & =-\frac{1}{N^{2} z} \ln |z| Q(0)+\ldots
\end{aligned}
$$

with the same equations for $Q^{+}$, except for a change of sign. These OPE's and the fact that $C(z)$ does not depend on $\bar{z}(\bar{\partial}\langle C(z) D(0)\rangle=0)$, enable us to identify $C, \bar{C}$ as generators of a continuous symmetry. It is this symmetry which is associated with the order parameter $\rho$.

Conformal field theories are characterized by their symmetry group and a number $\mathcal{C}$ called 'conformal charge'. Formally $\mathcal{C}$ is a coefficient in the pair correlation function of stress-energy tensor operators. A physical meaning of $\mathcal{C}$ becomes clear when we recall that a theory with an integer conformal charge $\mathcal{C}=k$ is equivalent to the theory with $k$ species of free bosonic fields. Thus $\mathcal{C}$ in unitary theories counts an effective number of degrees of freedom. The central charge of our theory is the sum of central charges of the free bosonic field $(\mathcal{C}=1)$ and the WZNW model on the $\mathrm{SU}(\mathrm{r})$ group:

$$
\mathcal{C}=1+\frac{N\left(r^{2}-1\right)}{N+r}=\frac{r}{N}+O\left(r^{2}\right)
$$

Thus the resulting central charge vanishes, as it must be; however, according to the definition of the replica limit (6) the physical correlation function of the stress-energy tensors remains finite:

$$
\langle T(z) T(0)\rangle=\lim _{r \rightarrow 0} \frac{2 N \mathcal{C}_{r}}{r} \frac{1}{2 z^{4}}=\frac{1}{z^{4}}
$$

Superficially this looks like the effective central charge $\mathcal{C}_{e f f}=2$. However, $\mathcal{C}_{\text {eff }}$ does not appear in the fusion rules of the stress-energy tensor components inside of correlation functions with matter fields, where we have

$$
T(z) T(\xi)=\frac{2}{(z-\xi)^{2}} T(\xi)+\frac{1}{z-\xi} \partial_{\xi} T(\xi)+\ldots
$$

As we have mentioned above, the numerical coefficient in (45) is fixed by the self-consistency requirements of $\mathrm{OPE}$.

Applying twice the OPE (39) to the four-point correlation function and using the Ward identities for $Q$ fields, we get the following set of identities: 


$$
\begin{aligned}
\langle T(z) C(1) D(2)\rangle & =\sum_{j=1,2}\left\{\frac{1}{\left(z-z_{j}\right)^{2}}+\frac{1}{z-z_{j}} \partial_{j}\right\}\langle C(1) D(2)\rangle \\
\langle\bar{T}(z) C(1) D(2)\rangle & =\sum_{j=1,2} \frac{1}{\bar{z}-\bar{z}_{j}} \bar{\partial}_{j}\langle C(1) D(2)\rangle=0 \\
\langle T(z) D(1) D(2)\rangle & =\sum_{j=1,2}\left\{\frac{1}{\left(z-z_{j}\right)^{2}}+\frac{1}{z-z_{j}} \partial_{j}\right\}\langle D(1) D(2)\rangle+\sum_{j=1,2} \frac{1}{z-z_{j}}\langle C(1) D(2)\rangle \\
\langle\bar{T}(z) D(1) D(2)\rangle & =\sum_{j=1,2}\left\{\frac{1}{\bar{z}-\bar{z}_{j}} \bar{\partial}_{j}\right\}\langle D(1) D(2)\rangle+\sum_{j=1,2} \frac{1}{\left(\bar{z}-\bar{z}_{j}\right)^{2}}\langle C(1) D(2)\rangle
\end{aligned}
$$

We can then substitute the two-point correlation functions to get

$$
\begin{aligned}
\langle T(z) C(1) D(2)\rangle & =-\frac{1}{2 N^{2}} \frac{1}{\left(z-z_{1}\right)^{2}\left(z-z_{2}\right)^{2}} \\
\langle T(z) D(1) D(2)\rangle & =\frac{2}{N^{2}} \frac{1}{\left(z-z_{1}\right)^{2}\left(z-z_{2}\right)^{2}}\left(\ln \left|z_{12}\right|-1 / 4\right) \\
\langle T(z) \bar{D}(1) \bar{D}(2)\rangle & =-\frac{1}{2 N^{2}} \frac{z_{12}^{2}}{\left(z-z_{1}\right)^{2}\left(z-z_{2}\right)^{2} \bar{z}_{12}^{2}}
\end{aligned}
$$

Taking into account Eq.(45) and Eqs.(42) we conclude that these expressions are compatible with the following OPE:

$$
\begin{aligned}
C(z) D(\xi, \bar{\xi}) & =-\frac{1}{2 N^{2}}\left[\frac{1}{(z-\xi)^{2}}+T(\xi)+\ldots\right] \\
D(z, \bar{z}) D(\xi, \bar{\xi}) & =\frac{2}{N^{2}}\left[\frac{\ln |z-\xi|}{(z-\xi)^{2}}+(\ln |z-\xi|-1 / 4) T(\xi)-\frac{(\bar{z}-\bar{\xi})^{2}}{4(z-\xi)^{2}} \bar{T}(\bar{\xi})+\ldots\right]
\end{aligned}
$$

and

$$
\begin{aligned}
T(z) C(\xi) & =\frac{C(\xi)}{(z-\xi)^{2}}+\frac{\partial_{\xi} C(\xi)}{(z-\xi)}+\ldots \\
T(z) D(\xi, \bar{\xi}) & =\frac{D(\xi, \bar{\xi})}{(z-\xi)^{2}}+\frac{C(\xi)}{(z-\xi)^{2}}+\frac{\partial_{\xi} D(\xi, \bar{\xi})}{(z-\xi)}+\ldots \\
\bar{T}(\bar{z}) D(\xi, \bar{\xi}) & =\frac{C(\xi)}{(\bar{z}-\bar{\xi})^{2}}+\frac{\partial_{\bar{\xi}} D(\xi, \bar{\xi})}{(\bar{z}-\bar{\xi})}+\ldots
\end{aligned}
$$

From the OPE's (43, 49), the Ward identity for the stress-energy tensor and primary fields and the Knizhnik-Zamolodchikov equation ( [12]) we derive the following Ward identity:

$$
\begin{aligned}
& \left\langle C\left(z_{1}\right) D\left(z_{2}, \bar{z}_{2}\right) Q\left(\xi_{1}, \bar{\xi}_{1}\right) \ldots Q^{+}\left(\xi_{2 N}, \bar{\xi}_{2 N}\right)\right\rangle \\
& =\frac{1}{2 N^{2}} \sum_{j} \frac{\sigma_{j}}{z_{1}-\xi_{j}}\left\langle C\left(z_{2}\right) Q\left(\xi_{1}, \bar{\xi}_{1}\right) \ldots Q^{+}\left(\xi_{2 N}, \bar{\xi}_{2 N}\right)\right\rangle-\frac{1}{2 N^{2} z_{12}^{2}}\left\langle Q\left(\xi_{1}, \bar{\xi}_{1}\right) \ldots Q^{+}\left(\xi_{2 N}, \bar{\xi}_{2 N}\right)\right\rangle
\end{aligned}
$$


where $\sigma=1$ for $Q$ and -1 for $Q^{+}$. Notice that the operator $D$ does not appear in the right hand side of this identity. This Ward identity is an important one since it, together with Eq.(49) establishes an isomorphism between the representations of the Virasoro algebra and the algebra of the conserved current $C$.

Now let us study the fusion of $Q$ with itself. For this end it is more convenient to rewrite the four-point correlation function (28) in terms of the solutions regular at $x \rightarrow \infty$. For Eq.(20) these solutions are $(N \neq 2)$

$$
\begin{aligned}
& \tilde{W}_{1}^{(0)}(x)=(-x)^{-1 / N} F(1 / N, 1 / N, 1+2 / N ; 1 / x), \\
& \tilde{W}_{2}^{(0)}(x)=(-x)^{-1 / N} F(1+1 / N, 1 / N, 1+2 / N ; 1 / x) \\
& \tilde{W}_{1}^{(1)}(x)=(-x)^{1 / N} F(-1 / N,-1 / N, 1-2 / N ; 1 / x), \\
& \tilde{W}_{2}^{(1)}(x)=-(-x)^{1 / N} F(1-1 / N,-1 / N, 1-2 / N ; 1 / x)
\end{aligned}
$$

These solutions have extremely simple monodromy properties:

$$
\begin{aligned}
& \tilde{W}_{1}^{(0)}(1-x)=\tilde{W}_{2}^{(0)}(x), \tilde{W}_{2}^{(0)}(1-x)=\tilde{W}_{1}^{(0)}(x) \\
& \tilde{W}_{1}^{(1)}(1-x)=-\tilde{W}_{2}^{(1)}(x), \tilde{W}_{2}^{(1)}(1-x)=-\tilde{W}_{1}^{(1)}(x)
\end{aligned}
$$

The crossing invariant form of the correlation function is

$$
W(x, \bar{x})=\alpha\left[\tilde{W}_{1}^{(0)}(x) \tilde{W}_{2}^{(0)}(\bar{x})-k^{2} \tilde{W}_{1}^{(1)}(x) \tilde{W}_{2}^{(1)}(\bar{x})+(x \rightarrow \bar{x})\right]
$$

where

$$
k=\frac{\Gamma(1+2 / N) \Gamma^{2}(-1 / N)}{\Gamma(1-2 / N) \Gamma^{2}(1 / N)}
$$

The coefficient $\alpha$ whose numerical value we do not provide should be choosen to match Eq.(58) to the correlation function (21) regular at $x=0$. Let us consider the limit $z_{31}=$ $\epsilon \rightarrow 0$ we have

$$
G(1,2 ; 1+0,2+0)=2 \alpha|\epsilon|^{-4 / N^{2}}\left[|z / \epsilon|^{4(N-2) / N^{2}}-k^{2}|z / \epsilon|^{-4(N+2) / N^{2}}\right]+\ldots
$$

This expansion is valid only for $N \neq 2$. In this case it corresponds to the standard operator product expansion: 


$$
Q(1) Q(2)=C_{1}^{1 / 2}\left|z_{12}\right|^{-4 \Delta+2 \Delta_{A}} O_{A}(2)+C_{2}^{1 / 2}\left|z_{12}\right|^{-4 \Delta+2 \Delta_{S}} O_{S}(2)+\ldots
$$

where $C_{1}, C_{2}$ are numerical coefficients and $O_{A}$ and $O_{S}$ are operators from the asymmetric and the symmetric representations whose Young tableaus are $(1,1,0, \ldots)$ and $(2,0, \ldots)$ respectively. Their conformal dimensions are given by Eq.(12):

$$
\Delta_{A}=\frac{2-N}{N^{2}}, \Delta_{S}=\frac{2+N}{N^{2}}
$$

which reproduces the result obtained in the previous publications [2] and [3].

At $N=2$ the dimension of the antisymmetric operator vanishes. Now we have a situation where there are three operators with zero conformal dimension - the unity, the adjoint operator and the operator in the antisymmetric representation. This situation will be discussed elsewhere.

\section{DEFORMATION BY THE LOGARITHMIC OPERATORS}

The conventional WZNW model remains an integrable theory even if one changes the coefficient in front of the $\operatorname{Tr}\left(\partial_{\mu} g^{+} \partial_{\mu} g\right)$-term in the action (4). According to [12], such per-

turbation is equivalent to the $J_{-1} \bar{J}_{-1} \Phi^{a b}$-operator (recall that $\Phi^{a b}$ is the primary field in the adjoint representation). The corresponding beta function is

$$
\beta(\gamma)=\frac{2 c_{v}}{c_{v}+N} \gamma
$$

where $\gamma$ is the deviation of the coupling constant from its critical value. In our case $c_{v}=r \rightarrow$ 0 and the beta function apparently vanishes. This means that the perturbation becomes marginal and we have to reconsider the terms of higher order in $\gamma$. Despite the fact that $\Phi^{a b}$ does not appear now in OPE, its decendants, that is the logarithmic operators $D, \bar{D}$ do appear. We suggest that the change in the coupling constant of the WZNW model (4) is associated with the perturbation by the marginal operator $\gamma \bar{D} D$. We warn the reader not to confuse this perturbation with a change of the disorder strength $\mathcal{A}$ which is truely irrelevant, leading to a change of the cut-off $M$. One physical mechanism of a marginal deformation 
away from criticality in the model (4) was described in [1] (see Chapter 7). We conjecture that this deformation is generated by the $\gamma \bar{D} D$-perturbation.

In the case of a deformation $\gamma \int \mathrm{d}^{2} z O(z, \bar{z})$ caused by a usual marginal operator $O$ one has two possibilities depending on the operator product expansion

$$
O(z, \bar{z}) O(0)=f \frac{O(0)}{|z|^{2}}+\ldots
$$

The first one is when $f=0$, i.e. the OPE of $O(z, \bar{z}) O(0)$ does not contain the operator $O$ itself. In this case this operator is truly marginal and one has the continuous family of conformal field theories parametrized by the deformation parameter (coupling constant). The anomalous dimensions $\Delta$ depend on this parameter. In the model (1) this situation is realized when one introduces an Abelian disorder (see [3]). In the opposite case, when $f \neq 0$, i.e. the OPE of $O(z) O(0)$ contains $O$ itself, there is a renormalization group (RG) flow of the coupling constant

$$
\frac{\mathrm{d} \gamma}{\mathrm{d} \ln \Lambda}=f \gamma^{2}+
$$

which means that the theory actually depends on the scale $\Lambda$.

Let us now study the same problem in a case where the theory is deformed by the operator $\bar{D} D$, which is truly marginal, because the OPE of $D(z) D(0)$ does not contain the operator $D$ itself (see Eq.(50). In this case we shall calculate the correlation function

$$
\begin{aligned}
G(z ; \gamma) & =\left\langle A(z) B(0) \exp \left(\gamma \int \mathrm{d}^{2} x \bar{D} D(x)\right\rangle\right. \\
& =\sum_{n} \frac{\gamma^{n}}{n !} \int\left\langle A(z) B(0) \bar{D} D\left(x_{1}\right) \ldots \bar{D} D\left(x_{n}\right)\right\rangle \mathrm{d}^{2} x_{1} \ldots \ldots \mathrm{d}^{2} x_{n}
\end{aligned}
$$

where $A$ and $B$ are some operators ( $Q$ and $Q^{+}$, for example) with the correlation function

$$
G(z ; 0)=\langle A(z) B(0)\rangle
$$

Using the OPE

$$
\bar{D} D(x) A(y)=a \frac{\ln ^{2}|x-y|^{2}}{|x-y|^{2}} A(y), \quad \bar{D} D(x) B(y)=b \frac{\ln ^{2}|x-y|^{2}}{|x-y|^{2}} B(y)
$$

one can find the first order in $\gamma$ correction to the correlation function which will be 


$$
\begin{aligned}
& \gamma \int\langle A(z) B(0) \bar{D} D(x)\rangle \mathrm{d}^{2} x= \\
& \quad a \gamma\langle A(z) B(0)\rangle \int \mathrm{d}^{2} x \frac{\ln ^{2}|x-z|^{2}}{|x-z|^{2}}+b \gamma\langle A(z) B(0)\rangle \int \mathrm{d}^{2} x \frac{\ln ^{2}|x|^{2}}{|x|^{2}}
\end{aligned}
$$

where in both integrals we integrate over $x$ between 0 and $z$. Then it is easy to find the following logarithmic correction:

$$
\gamma \frac{(a+b)}{3} \ln ^{3}|z|^{2} G(z ; 0)
$$

Now one can consider the next order corrections and sum all of them using the same methods as in the case of conventional marginal operators (see [14]). The result is

$$
G(z ; \gamma)=G(z ; 0) \exp \left(\gamma \frac{a+b}{3} \ln ^{3}|z|^{2}\right)
$$

which is different from the case of a conventional marginal operator when one has the first power of $\log$ in the exponent and not the third. The first power in the exponent introduces the power factor

$$
\exp \left((a+b) \gamma \ln |z|^{2}\right)=|z|^{2(a+b) \gamma}
$$

corresponding to the change in the anomalous dimension $\Delta_{A}(\lambda)=\Delta_{A}(0)-(a+b) \gamma$, and the behaviour of the deformed correlation function is still power-like. The are no logarithmic corrections after all. This is not true anymore with the logarithmic operator, when the correlation function cannot be written as a power at all. Thus we see that the correlation functions for operators which have non-trivial OPE with the logarithmic operator $D$ (like our primary fields $Q$, for example) will have logarithmic corrections in the deformed theory - even in the absence of the RG flow.

Let $A=Q$ and $B=Q^{+}$, then, according to Eq.(43), $a=b=N^{-4}$. At $\gamma<0$ the correlation function (66) decays faster then any power. At $\gamma>0$ it increases faster than any power. In this case the approximation leading to Eq.(66) breaks down when the correlation function begins to increase, that is at

$$
|z| \sim M^{-1} \exp \left[\frac{N}{4 \sqrt{\gamma}}\right]
$$


We speculate that for $\gamma>0$ the symmetry is broken and the finite density of states at $\epsilon=0$ is formed. This probably explains the finite DOS obtained numerically in disordered d-wave superconductors by Wheatley [15].

\section{PROBABILITY DISTRIBUTION OF LOCAL DOS}

Now we shall calculate the distribution function of local densities of states. We can do it for a system of a finite size $L$. From Eq.(5) we know that in the case of zero frequency we have

$$
\left\langle\rho^{n}(x)\right\rangle=M^{n}\left\langle\left[\operatorname{Tr}\left(Q+Q^{+}\right)\right]^{n}\right\rangle \sim L^{-2 \Delta_{n}}
$$

The latter equality is valid in the leading order in $1 / L ; \Delta_{n}$ is given by Eq.(13) being the smallest conformal dimension in the operator product of $\mathrm{n}$ operators $\operatorname{Tr}\left\langle\left(Q+Q^{+}\right)\right\rangle$. For $N>2 \Delta_{n}$ 's are negative for $n>1$.

Let us imagine now that the result (69) comes from a local distribution function of $\rho$ :

$$
\left\langle\rho^{n}(x)\right\rangle=\int_{0}^{\infty} P(\rho) \rho^{n} \mathrm{~d} \rho=A_{n} \exp \left\{2 \ln L\left[\frac{(N-1) n^{2}}{2 N^{2}}-\frac{n}{2 N}\right]\right\}
$$

where $A_{n}$ may contain powers of $\ln L$. The distribution function which reproduces this

result is the famous log-normal distribution which is considered as a characteristic feature of disordered systems [16], [17], [19]:

$$
\begin{array}{r}
P(\rho)=D(\rho) \exp \left[-\frac{1}{\ln L^{\eta}} \ln ^{2}\left(\rho L^{\zeta}\right)\right], \\
\zeta=\frac{1}{N}(3-2 / N), \eta=\frac{4(N-1)}{N^{2}}
\end{array}
$$

where $D(\rho)$ is a smooth function of $\ln \rho$ which we cannot determine.

From the fact that the frequency scales as $L^{-2+2 \Delta_{1}}$ we can conjecture that the distribution function of $\rho(\omega)$ is given by

$$
\begin{array}{r}
P\left(\rho_{\omega}\right)=D\left(\rho_{\omega}\right) \exp \left[-\frac{1}{\ln \left(1 / \omega^{\gamma}\right)} \ln ^{2}\left(\rho_{\omega} \omega^{-\beta}\right)\right], \\
\beta=\frac{3 N-2}{2 N^{2}-1}, \gamma=\frac{4(N-1)}{2 N^{2}-1}
\end{array}
$$


The authors of Ref. [3] have discussed this distribution without writing it down explicitly.

It is worth remarking that the log-normal distribution also appears in the Liouville theory (27). Indeed, according to Eq.(32), the conformal dimension of the operator $\exp (n \alpha \phi)$ is given by $\frac{1}{2} n \alpha(Q-n \alpha)$, i.e. it has the same quadratic $n$-dependence as $\Delta_{n}$ (13). Repeating the previous arguments we obtain for the vertex operator $V=\exp (\alpha \phi)$ the log-normal distribution (71) with $\eta=4 \alpha^{2}, \zeta=\alpha Q(3-2 \alpha Q)$. Since the logarithmic operators appear in this theory only when $Q=2 \alpha$, we conclude that their presence is not directly related to multifractality of the target space.

\section{CONCLUSIONS}

In this paper we have demonstrated that in the general class of nonunitary critical models a new phenomenon takes place - the emergence of logarithmic operators associated with a special hidden continuous symmetry. The presence of this symmetry is intimately related to the fact that the order parameter of our model - the local density of states at $\epsilon=0$ - does not acquire a non-zero average. Since above mentioned features appear also in quantum gravity [10], we anticipate a connection between quantum gravity and critical models with disorder [18]. Our expectations are supported by the recently discovered similarities between the conventional localization theory and the Liouville theory [19], [20].

The physical meaning of the hidden symmetry in models with disorder as well as in $2 \mathrm{~d}$ gravity, remains obscure. It is clear, however, that this symmetry should routinely appear in critical non-unitary theories where the Hamiltonian cannot be diagonalized (in our paper this fact is expressed in Eq.(34).

It follows also from our work that, at least for the model in question, the replica approach is equivalent to the supersymmetric one. This is a pleasant fact.

As we have said above, Eqs.(49) and (55) enable one in principle to reformulate the theory in terms of representations of the current algebra of the conserved current $C(z)$. With this task being accomplished one can abanbon replicas and treat the theory axiomatically as it 
is customary, for instance, in the theory of the standard WZNW model. At present this remains the biggest challenge.

\section{ACKNOWLEDGMENTS}

The authors are grateful to B. Altshuler, J. Chalker, K. Efetov, V. Fal'ko, A. Nersesyan, D. Khmelnitskii, N. Mavromatos, B. Muzykantskii, R. Stinchcombe and J. Wheater for valuable and inspirational discussions. Our special thanks are to I. Lerner, who acquainted us with the results obtained in the conventional localization theory. One of us (I.I.K) would

like also to thank A. Bilal and V. Gurarie for numereous interesting discussions about the logarithmic operators.

\section{Appendix}

In this Appendix we write down the relationship between conformal blocks in the replica and the supersymmetric (SUSY) representations. According to Ref. [3], the correlation functions in SUSY representation are products of correlation functions of the Gaussian model and the $\mathrm{SU}_{k}(\mathrm{~N})$ WZNW theory with $k=-2 N$. According to Ref. [12], the conformal blocks of the latter model are

$$
\begin{aligned}
& W_{1}^{(0)}(x)=(1-x)^{-1} F(1 / N,-1 / N, 2 ; x) \\
& W_{2}^{(0)}(x)=-\frac{1}{2 N} x(1-x)^{-1} F(1+1 / N, 1-1 / N, 3 ; x)
\end{aligned}
$$

The second solutions contain logarithms. The relationship between two representations is established by the identity

$$
\begin{array}{r}
x\left(1-1 / N^{2}\right) F(1+1 / N, 1-1 / N, 3 ; x)+2(1-x) F(1 / N,-1 / N, 2 ; x) \\
=\left(1-x N^{2}\right) F(1 / N,-1 / N, 1 ; x)
\end{array}
$$

using which one can write the expression for four-point function (18) in terms of conformal blocks either of replica or supersymmetric models. 


\section{REFERENCES}

[1] A. A. Nersesyan, A. M. Tsvelik and F. Wenger, Phys. Rev. Lett. 72, 2628 (1994); Nucl. Phys. B.438, 561 (1995).

[2] A. M. Tsvelik, Phys. Rev. B51, 9449 (1995).

[3] C. Mudry, C. Chamon, X.-G. Wen, unpublished.

[4] R. Gade, private communication.

[5] K. B. Efetov, Adv. Phys. 32, 53 (1983).

[6] This statement is usually called the Mermin-Wagner theorem. In non-unitary theories which admit operators with non-positive scaling dimensions such operators may acquire non-zero averages. However, this does not concern the operator $Q$ whose scaling dimension is positive.

[7] L. P. Gor'kov and P. A. Kalugin, Pis'ma v ZhETP41, 208 (1985) [JETP Lett. 41253 (1985)].

[8] L. Rozansky and H. Saleur, Nucl. Phys. B376, 461 (1992).

[9] V. Gurarie, Nucl. Phys. B410, 535 (1993); hep-th/9303160.

[10] A. Bilal and I.I. Kogan, PUPT-1482, hep-th@xxx/9407151 (unpublished); Nucl. Phys. B449, 569 (1995); hep-th@xxx/9503209.

[11] J. Polchinski, Nucl. Phys. B346, 253 (1990); Nucl. Phys. B357, 241 (1991).

[12] V.G. Knizhnik and A. B. Zamolodchikov, Nucl. Phys. B247, 83 (1984).

[13] Handbook of Mathematical Functions, Ed. by M. Abramovitz and I. A. Stegun, ed. by National Bureau of Standards, (1964).

[14] V. L. Pokrovskii and B. Patashinskii, Fluctuations Theory of Phase Transitions (in Russian), Moscow, 1975. The method used in this book is due to 
A. M. Polyakov, Sov. Phys. ZhETF 63, 24 (1972).

[15]. J. Wheatley, preprint.

[16] B. Altshuler, V. Kravtsov and I. Lerner, Sov. Phys. JETP Lett. 43, 441; Sov. Phys. JETP 64, 1352 (1986); Phys. Lett. A134, 488 (1989).

[17] I. Lerner, Phys. Lett. A133, 253 (1988).

[18] I. I. Kogan and A.M. Tsvelik, work in progress.

[19] K. B. Efetov and V. I. Fal'ko, preprint.

[20] D. E. Khmelnitskii and B. A. Muzykantskii, preprint. 